\title{
Editorial
}

\section{Pulmonary Function Test So Informative yet Underutilized}

\author{
Mohammed S. Mateenuddin ${ }^{1}$ \\ ${ }^{1}$ Department of Pulmonology, Intensivist Sunshine Hospitals, \\ Hyderabad, India \\ Ind J Car Dis Wom:2020;5:297-298
}

Pulmonary function tests are not just spirometry! It includes a gamut of other evaluations like lung volumes, diffusing capacity of lung for carbon monoxide (DLCO), airway resistance and lung compliance, evaluation of respiratory muscle functions, central respiratory drive, polysomnography (sleep study), and in advance setup, comprehensive evaluation by cardiopulmonary exercise testing (CPET or CPX) and nutritional assessment by performing indirect calorimetric studies. All these when judiciously utilized are immensely helpful not only for pulmonary disorders but also for evaluation, management and follow-up of concomitant disorders of cardiovascular system, central nervous system, musculoskeletal system, renal diseases, gastrointestinal diseases, collagen vascular diseases, and endocrine and metabolic disorders. The article in this journal by Kommu et al (REFERENCE) attempted to correlate declining lung function, especially severity of obstructive defect in postmenopausal women with pulse pressure and arterial stiffness, which definitely needs further properly designed evaluation.

In a similar earlier study by Wu et al, increased arterial stiffness was linked to restrictive spirometry pattern in both men and women, with decreased FVC \& FEV ${ }_{1}$ with normal or increased $\mathrm{FEV}_{1} / \mathrm{FVC}$ ratio. ${ }^{1}$

Chronic obstructive pulmonary disease (COPD) broadly constitutes the following three afflictions: 1 . pulmonary emphysema (enlargement of airspaces with destruction of alveolar walls, formation of bullae, loss of elastic recoil and airways obstruction); 2 . chronic bronchitis (which has a clinical definition: having cough with expectoration for at least 3 months for 2 consecutive years); and 3. small airways disease (disease involving the small bronchioles).

Fair amount of differentiation on these three can be done noninvasively if appropriate lung function testing including spirometry, lung volumes (body plethysmography), DLCO and tests for airways resistance and lung compliance are judiciously performed and interpreted, which are immensely helpful in the management, follow-up and prognostication.

Address for correspondence Mohammed S. Mateenuddin, MBBS, DTRD, DNB, MNAMS, Department of Pulmonology, Intensivist Sunshine Hospitals, Hyderabad, India (e-mail: matintensivist@gmail.com).
The chronic inflammation in COPD is mainly due to smoke and other chronic irritants which amplify the normal inflammatory response of the airways. The mechanism by which this amplification occurs is not fully understood, having a genetic predisposition in quite likely. The inflammatory mediators and the cells involved in this amplification of inflammatory response are responsible the local effects as well as for the systemic effects of the inflammation. The cellular components of inflammation are neutrophils, eosinophils, lymphocytes and macrophages.

The inflammation in COPD is seen at pulmonary level as well as at the systemic level. Thus, COPD might be considered a disease mainly involving the lungs but have extensive systemic manifestations.

Regarding hyperinflation, Vassilakopoulos et al have shown that though the cytokine production is stimulated at pulmonary level, it significantly contributes to the systemic inflammation. $^{2}$

The skeletal muscles (loss of muscle mass) and the bone marrow (anemia) are apparently affected due to systemic inflammation in COPD. Thus, Rabinovich et al found systemic inflammation reflected by the TNF- $\alpha$ levels in plasma. This increases after muscular exercise in COPD patients but not in healthy subjects. ${ }^{3}$

Heart diseases and pulmonary diseases have mostly similar symptomatic presentation; even in grading of symptoms, they have many common risk factors and similarities. New York Heart Association (NYHA) heart failure grading, modified Medical Research Council (mMRC), Dyspnoea Grading, and Canadian Cardiovascular Society (CCS) angina grading have quite a few similarities.

Presence of comorbidities involving other systems, especially cardiovascular system (CVS), are recognized with most of the respiratory disorders such as COPD, asthma, ${ }^{4}$ bronchiectasis, lung cancer and diffuse lung diseases (interstitial lung diseases [ILDs]), affecting the health-related quality of life (HRQOL), while increasing the morbidity and
DOI https://doi.org/ 10.1055/s-0040-1722555.

published online December 31, 2020
This is an open access article published by Thieme under the terms of the Creative Commons Attribution-NonDerivative-NonCommercial-License, permitting copying and reproduction so long as the original work is given appropriate credit. Contents may not be used for commercial purposes, or adapted, remixed, transformed or built upon. (https://creativecommons.org/licenses/by-nc-nd/4.0/).

Thieme Medical and Scientific Publishers Pvt. Ltd. A-12, 2nd Floor, Sector 2, Noida-201301 UP, India 
mortality. It is accepted as a part of the cardiopulmonary continuum. ${ }^{5}$ Therefore, early and correct recognition of dysfunctions with appropriate multispecialty management requires precise evaluation by performing the appropriate lung function tests in addition to spirometry, ${ }^{6}$ in order to optimize and economize the usage of healthcare resources while improving HRQOL and survival.

Patients with respiratory diseases, especially COPD, have a significantly high cardiovascular morbidity and mortality independent of the common risk factors. Presence of higher levels of inflammation markers, even in stable pulmonary disease when there is no exacerbation, indicates a link between systemic inflammatory processes and the local pulmonary inflammation. Hence, anti-inflammatory therapy with inhaled corticosteroids (ICS) also modulates systemic inflammation and is found to be beneficial. In COPD, a decrease in hyperinflation over stretching of the tissues and relative improvement in respiratory mechanics were associated with a reduction in systemic inflammation and thereby cardiovascular morbidity, which strongly suggests the need for proper assessment of lung volumes to know the entrapment of air or hyperinflation in order to adjust the treatments.

Pleiotropic immune modulatory and anti-inflammatory effects are seen with Statins, in addition to the commonly accepted use for reduction of serum cholesterol levels. In approximately $80 \%$ of patients undergoing coronary intervention concomitant, COPD is not appreciated. This usually happens in early to moderate stages, where appropriate management of associated comorbidities will significantly reduce the mishaps and morbidity. Similarly, previous myocardial infractions are not appreciated by clinicians in $70 \%$ of patients presenting with acute COPD exacerbations, although ECG signs are present if seen thoroughly. Beta blockers are the mainstay of drug treatment in stable coronary heart disease (CHD), which definitely helps with alleviation of symptoms and improved prognosis, but many a times, these medications are withheld or underdosed in COPD patients, as the physicians are unnecessarily apprehensive of the side effects, that is, bronchoconstriction and aggravation of breathlessness, which is definitely of concern after myocardial infarction. ${ }^{7}$ Cardioselective $\beta$ blockers are relatively safe when partially reversible obstruction is present. Renin-angiotensin-aldosterone system (RAAS) inhibitors are many a times the choice in patients with heart failure, arterial hypertension or diabetes mellitus to reduce morbidity and mortality. They are also assumed to have beneficial effects on systemic inflammation, causing improvement in skeletal muscle function, peripheral oxygen consumption, and erythrocytopoitic system, reflecting the necessity of detailed evaluation of pulmonary functions. While acetylsalicylic acid causes an increased risk of exacerbation of asthma, reversible $\mathrm{P}_{2} \mathrm{Y}_{12}$-antagonists (ticagrelor, cangrelor, and elinogrel) may lead to increased dyspnea and respiratory insufficiency. In contrast, irreversible substances such as clopidogrel and prasugrel do not seem to be associated with increased respiratory symptoms. Pulmonary function evaluation and the evaluation of cardiac limitations should only be done in stable, euvolemic phases of about 3 months' time frame. Both organ systems should be compulsorily included in diagnostic considerations. ${ }^{8}$ This holds especially relevant as noninvasive diagnostic tools as well as treatment strategies are widely available. In approximately $20 \%$ of COPD patients, left heart failure is diagnosed for the first time after extensive cardiovascular workup; vice versa, obstructive ventilation disorders are diagnosed or appreciated for the first time in greater than $30 \%$ of heart failure patients on proper evaluation. However, poor acoustic window may be present in approximately $50 \%$ of these patients, depending on the degree of airflow limitation. Evaluation of heart failure with preserved ejection fraction (HFpEF) is particularly important and difficult in this setting and associated with false diagnoses due to pulmonary comorbidity. Alternatives include cardiac magnetic resonance imaging as well as CPET or CPX.

Hypoxia causes increased inflammation, sympathetic activity and blood viscosity among other factors, and it is likely to be responsible for the underlying pathogenesis. Sleep-disordered breathing (SDB) has been associated and partially responsible in multiple CV diseases including hypertension, ischemic heart disease, congestive heart failure, arrhythmias, and stroke. However, to what extent the SDB, including obstructive and central sleep apneas, is responsible for the development of cardiovascular diseases is not fully understood. ${ }^{9}$

Properly requisitioned, performed and interpreted pulmonary function tests can change the management approach and prognosis of various chronic diseases, especially of the CVS. Therefore, future diagnostic and therapeutic strategies should be guided by an integrative approach and precise phenotyping of the disease entities.

\section{Conflicts of Interest}

None declared.

\section{References}

$1 \mathrm{Wu} \mathrm{IH}$, Sun ZJ, Lu FH, et al. Restrictive spirometry pattern is associated with increased arterial stiffness in men and women. Chest 2017;152(2):394-401

2 Hamid Q, Shannon J, Martin J, Physiologic Basis of Respiratory Disease. Montreal: McGill University; 2005

3 Rabinovich RA, Figueras M, Ardite E, et al. Increased tumour necrosis factor- $\alpha$ plasma levels during moderate-intensity exercise in COPD patients. Eur Respir J 2003;21(5):789-794

4 Alonso-Gonzalez R, Borgia F, Diller GP, et al. Abnormal lung function in adults with congenital heart disease: Prevalence, relation to cardiac anatomy, and association with survival. Circulation 2002;106:2555-2560

5 Trinkmann F, Saur J, Borggrefe M, Akin I. Cardiovascular comorbidities in COPD. J Clin Med 2019;8(1):69

6 Ramalho SHR, Shah AM. Lung function and cardiovascular disease: a link. Trends Cardiovasc Med 2020;(e-pub ahead of print). doi: https://doi.org/10.1016/j.tcm.2019.12.009

7 Cardiovascular Comorbidities in COPDJ. Clin Med (Lond) 2019;10

8 Trends Cardiovasc Med Erikson B. 2020

9 Chowdhuri S, Crook ED, Taylor HA, Jr, Badr MS. Cardiovascular complications of respiratory diseases. Am J Med Sci 2007;334(5):361-380 